\title{
Crowdfunding en tiempos de la COVID-19
}

\author{
Crowdfunding in times of COVID-19 \\ CIRIEC-UNED 2021-050-054 \\ DOI: $10.22458 /$ rna.v 12i2.3864
}

Ph.D. Cinta Borrero-Domínguez

Departamento de Economía Financiera y Dirección de Operaciones

Universidad de Sevilla, España

cborrero2@us.es

orcid.org/0000-0001-8216-6239

Ph.D. Encarnación Cordón-Lagares

Departamento de Economía

Universidad de Huelva, España

ecordon@uhu.es.

orcid.org/0000-0002-8677-5960
Ph.D. Rocío Hernández-Garrido

Departamento de Dirección de Empresas y Marketing

Facultad de Ciencias Empresariales y Turismo

Universidad de Huelva, España

rocio.hernandez@dem.uhu.es

orcid.org/OOOO-0001-7527-9809

\section{RESUMEN:}

Este estudio se ha centrado en la plataforma GoFundMe, la cual es considerada como la que tiene un mayor volumen de campañas relacionadas con la COVID-19. GoFundMe es una plataforma que se encuadra dentro de la modalidad de crowdfunding de donación. El principal objetivo de este trabajo consiste en el análisis de las campañas de crowdfunding relacionadas con la COVID-19, que se han recopilado de manera manual de la plataforma de GoFundMe, con el propósito de llevar a cabo una descripción de las características de una muestra aleatoria de 118 proyectos. Mediante la utilización de diferentes técnicas del análisis estadístico de tipo descriptivo se analizan un conjunto de variables cuantitativas, como son la tipología del proyecto, importe recaudado, importe objetivo, y estadísticas sobre la recaudación de los fondos relativa al número de donantes, veces compartidos y seguidores, entre otras.

\begin{abstract}
:
This study has focused on the GoFundMe platform, which is considered to have the highest volume of campaigns related to COVID-19. GoFundMe is a platform that falls within the donation crowdfunding modality. The main objective of this paper consists of the analysis of the crowdfunding campaigns related to COVID-19, which have been collected manually from the GoFundMe platform, in order to carry out a description of the characteristics of a sample random of 118 projects. Through the use of different techniques of descriptive statistical analysis, a set of quantitative variables are analyzed, such as the type of project, amount collected, target amount, and statistics on fundraising related to the number of donors, times shared and followers, among others.
\end{abstract}

\section{RÉSUMÉ :}

Cette étude se concentre sur la plateforme GoFundMe, qui est considérée comme celle qui a le plus de campagnes liées à la COVID-19. GoFundMe est une plate-forme de financement participatif. Le principal objectif de ce travail est d'analyser les campagnes de financement participatif liées à la COVID-19, qui ont été compilées manuellement à partir de la plate-forme GoFundMe, en vue d'une description des caractéristiques d'un échantillon aléatoire de 118 projets. Différentes techniques d'analyse statistique descriptive permettent d'analyser un ensemble de variables quantitatives, telles que la typologie du projet, le montant collecté, le montant cible et les statistiques sur le nombre de donateurs collectés, fois partagés et adeptes, entre autres.

\section{RESUMO:}

Este estudo foca-se na a plataforma GoFundMe, a qual é considerada a de maior volume de campanhas relacionadas à COVID-19. GoFundMe é uma plataforma que se enquadra na modalidade de crowdfunding de doação. $O$ objetivo principal deste trabalho consiste na análise das campanhas de crowdfunding relacionadas com a COVID-19, recolhidas manualmente na plataforma GoFundMe, com o propósito de realizar a descrição das características de uma amostra aleatória de 118 projetos. Por meio da análise estatística descritiva são analisadas um conjunto de variáveis quantitativas, como a tipologia do projeto, valor arrecadado, valor objetivo e estatísticas sobre a arrecadação dos recursos em relação ao número de doantes, vezes compartilhado e seguidores, entre outras.
MOTS-CLÉS :

CROWDFUNDING,

FONDATEURS, ECONOMIE

SOCIALE, COVID-19.
PALAVRAS-CHAVES:

CROWDFUNDING,

FUNDADORES, ECONOMIA

SOCIAL, COVID-19. 


\section{INTRODUCCIÓN}

A finales del primer trimestre del año 2020, la llegada vertiginosa de la pandemia de la COVID-19 empezó a avecinar una gran tormenta económica y social a nivel mundial, cuyos efectos, están ocupando un lugar importante en los debates públicos, y sobre todo en los trabajos de investigaciones académicas.

En tiempos difíciles, donde la pandemia, desencadena en una crisis sanitaria, económica y social sin precedentes, el instinto de las personas por ayudar a los demás de alguna manera, hizo que hubiera un aumento significativo de campañas benéficas (Farhoud et al., 2021), en busca de una financiación colectiva ante unas consecuencias devastadoras e impredecibles que se estaba presentando de forma muy rápida.

El crowdfunding, por tanto, ha sido y es una importante herramienta de financiación (Rajwa et al., 2020) y un método exitoso de recaudación de fondos, que juega un papel importante, para apoyar numerosas causas médicas y sociales, entre ellos, las relacionadas con la COVID-19 (Renko et al., 2019), ya que, a medida que la emergencia sanitaria se extendía rápidamente por los países, surgieron muchas campañas de crowdfunding para solventar la situación (Merelli, 2020).

La plataforma en línea GoFundMe, objeto de nuestro estudio, es caracterizada por su fácil accesibilidad y cuenta con un gran número de proyectos, donde se puede observar un aumento paulatino y significativo de los proyectos relacionados con la COVID-19.

Tal y como afirma Farny et al. (2019), el apoyo de los emprendedores sociales, en tiempos de crisis, es una fuente esencial para cubrir la falta de apoyo gubernamental, necesarios para una recuperación social y económica de los agentes implicados, ya que han sido muchas las personas desfavorecidas que se han encontrado indefensas y sin medios para sobrellevar la delicada situación en la que se han vistos inmersas.

La pandemia de la COVID-19, ha demostrado que un gran número de empresas sociales han intentado adaptarse y sobrevivir, enfrentándose para ello, a los importantes desafíos que han venido provocados por una lucha constante y activa frente a problemas sociales causados por la pandemia.

Más allá de mitigar el efecto de la crisis provocada por la pandemia, es probable que el crowdfunding proporcione una señal temprana de las necesidades emergentes y el sentimiento social de las comunidades en grave peligro (Saleh et al., 2021).

Tal y como afirma Cadogan (2021) el crowdfunding relacionado con el coronavirus ha sufrido un aumento exponencial importante corroborándose con la plataforma objeto de estudio como es GoFundMe, la cual ha recibido un alto número de solicitudes (Elmer et al., 2020) y de donde se pueden extraer un gran número de proyectos de economía colaborativa con respecto a la COVID-19 a nivel mundial durante el brote de la pandemia.

La plataforma reseñada, empezó a tener un funcionamiento muy intenso y un uso desconocido hasta la fecha, en los primeros meses de la conocida crisis sanitaria (Ryssdal et al., 2020).

\section{DESCRIPCIÓN DE LOS DATOS Y METODOLOGÍA}

La información necesaria para realizar el estudio se ha obtenido a partir de una muestra aleatoria de 118 proyectos de crowdfunding relacionados con COVID-19 recopilados de la plataforma GoFundMe.

Como se muestra en la Tabla 1, las modalidades de proyectos de crowdfunding que se han creado con más frecuencia son los proyectos que pertenecen a la categoría "Médicas, enfermedades y salud" con el $48 \%$ de proyectos, seguido de la categoría de "Accidentes y emergencias" con el $11 \%$ de los mismos. Estos resultados son como se esperaban debido a que un gran porcentaje de las campañas de crowdfunding se han creado para solventar la difícil situación que ha provocado la emergencia sanitaria provocada por la COVID-19.

En lo que respecta al importe recaudado, las modalidades de proyectos que presentan unos mayores importes medios es la de "Comunidad y vecinos" con un importe medio recaudado superior a los cinco millones de euros, seguido por la categoría de "Accidentes y emergencias" con un valor medio cercano al millón de euros. En este sentido, cabe indicarse que el importe medio recaudado para el total de proyectos ha sido de aproximadamente los 532.000 euros.

Por otra parte, el mayor número medio de donantes se encuentran en la categoría de "Accidentes y emergencias" con un valor medio de 17.330 donantes por proyecto, seguidos por las categorías de "Comunidad de vecinos" y "Medioambiente" con unos valores medios de 9.437 y 6.800 donantes por proyecto, respectivamente. En cuanto al valor medio del número de donantes para el total de categorías seleccionadas ha sido de 4.365. 
Por último, respecto a las variables cuantitativas número de veces que el proyecto es compartido mediante las redes sociales y número de seguidores de los proyectos, las categorías que presentan unos mayores valores medios coinciden con las categorías que presentaban un mayor número medio de donantes, la cuales son las categorías de "Accidentes y emergencias" (22.673 veces compartido y 17.283 seguidores por proyecto), "Comunidad de vecinos" (11.048 veces compartido y 9.473 seguidores por proyecto) y "Medioambiente" (8.500 veces compartido y 6.700 seguidores por proyecto). En este caso, el número medio de veces en el que el proyecto es compartido y el número medio de seguidores para el total de los 118 proyectos es de 5.601 y 4.325, respectivamente.

Tabla 1. Características de los proyectos por categoría

\begin{tabular}{|c|c|c|c|c|c|c|}
\hline Tipo de proyecto & $N^{\circ}$ & $\begin{array}{c}\text { Importe medio } \\
\text { recaudado (en } \\
\text { euros) }\end{array}$ & $\begin{array}{c}\text { Importe } \\
\text { medio } \\
\text { objetivo (en } \\
\text { euros) }\end{array}$ & $\begin{array}{l}\mathrm{N}^{\circ} \\
\text { medio de } \\
\text { Donantes }\end{array}$ & $\begin{array}{c}\mathrm{N}^{\circ} \text { medio } \\
\text { de veces } \\
\text { compartido }\end{array}$ & $\begin{array}{l}\mathrm{N}^{\circ} \\
\text { medio de } \\
\text { seguidores }\end{array}$ \\
\hline Accidentes y emergencias & 13 & 1.009 .147 & 1.773 .552 & 17.330 & 22.673 & 17.283 \\
\hline $\begin{array}{l}\text { Alquiler, comida y facturas } \\
\text { mensuales }\end{array}$ & 4 & 6.969 & 34.894 & 142 & 344 & 141 \\
\hline Animales y mascotas & 1 & 25.000 & 25.000 & 903 & 862 & 898 \\
\hline Artes creativas, música y cine & 3 & 56.914 & 118.689 & 776 & 1.722 & 773 \\
\hline Bebés, niños y familias & 3 & 112.769 & 115.508 & 946 & 1.979 & 947 \\
\hline Celebraciones y eventos & 1 & 22.811 & 25.134 & 237 & 729 & 234 \\
\hline Comunidad y vecinos & 8 & 5.230 .643 & 5.456 .520 & 9.437 & 11.048 & 9.473 \\
\hline Deportes, equipos y clubes & 1 & 4.457 & 5.865 & 76 & 438 & 76 \\
\hline Funerales y recordatorios & 7 & 12.600 & 11.250 & 221 & 779 & 221 \\
\hline Médicas, enfermedades y salud & 57 & 91.751 & 138.029 & 3.019 & 3.591 & 2.945 \\
\hline Medioambiente & 1 & 550.650 & 628.352 & 6.800 & 8.500 & 6.700 \\
\hline Negocios y emprendedores & 6 & 29.041 & 55.073 & 531 & 1.447 & 546 \\
\hline $\begin{array}{l}\text { ONGs y organizaciones } \\
\text { benéficas }\end{array}$ & 3 & 50.993 & 83.453 & 1.062 & 1.295 & 986 \\
\hline Otras & 6 & 75.688 & 165.795 & 1.283 & 4.551 & 1.283 \\
\hline Sueños, esperanzas y deseos & 1 & 30.429 & 83.780 & 1.900 & 195 & 1.900 \\
\hline Voluntariado y servicios & 3 & 177.162 & 200.894 & 3.653 & 1.530 & 3.687 \\
\hline
\end{tabular}

Fuente: Elaboración propia a partir de los datos recopilados de la plataforma GoFundMe

Respecto a los proyectos que han resultado exitosos porque han conseguido el importe objetivo, cabe indicarse que del total de los 118 proyectos analizados únicamente el 19,49 \% de los mismos consiguió el importe objetivo establecido a la hora de su creación. No obstante, si el análisis se lleva a cabo por categoría de proyectos se observa que los mayores porcentajes de éxito se encuentran en las categorías de "Alquiler, comida y facturas mensuales", "Funerales y recordatorios", "Comunidad de vecinos" y "Artes creativas, música y cine" con el 50 \%, 42,86 \%, 37,5\% y $33,33 \%$, respectivamente.

\section{Conclusiones y futuras líneas de investigación}

El crowdfunding de proyectos socialmente comprometidos, es y será una pieza importante de un puzzle que nos ayudará a conseguir una estrategia más sostenible y llevadera en el futuro post pandémico que nos espera.

En esta fuente de financiación alternativa, tendrán cabida proyectos relacionados con la COVID-19 de todas las categorías, tal y como se puede apreciar en la estadística anteriormente expuesta. 
Por ello, es necesario seguir avanzando en las variables que pueden influir a la hora de que una campaña sea exitosa o no. Aunque, se trata de un tema ampliamente estudiado en la literatura previa, es fundamental aplicar ese tipo de estudios a proyectos específicamente relacionados con la COVID-19 con objeto de averiguar si las variables que han sido previamente consideradas como las que influyen en el éxito también siguen siéndolo en este tipo de proyectos. En este sentido, el estudio ampliará el análisis estadístico con métodos econométricos en el que se emplearán variables cuantitativas y cualitativas, con objeto de establecer relaciones significativas entre las mismas.

La pandemia de la COVID-19, junto a la incertidumbre, la interrupción y el surgimiento de nuevas y urgentes necesidades está obligando a los investigadores de emprendimiento social a mirar más allá de los conflictos y tensiones organizacionales, y considerar el "panorama general" que incluye esfuerzos para abordar los problemas sociales globales (Bacq, S., Lumpkin, 2020).

A diferencia de la mayoría de los desastres, los cuales, generalmente tienen una fase aguda de destrucción y luego una de recuperación, los impactos de la pandemia sobre la economía y salud serán duraderos, y esto se refleja en el prolongado crecimiento de campañas de crowdfunding (Igraa et al., 2021).

Además, se puede afirmar, tal y como se desprende de los resultados obtenidos, el compromiso de la sociedad ante la situación vivida en estas fechas lo que se traduce en un refuerzo de las aportaciones dedicadas a proyectos relacionados con accidentes y emergencias y de comunidad y vecinos.

Esto, pone de manifiesto la ola de solidaridad que se ha producido a raíz de la pandemia provocada por la crisis sanitaria de la COVID-19. 


\section{REFERENCIAS}

Bacq, S., Lumpkin, G. 2020. Social entrepreneurship and COVID-19. Journal of Management Studies, forthcoming.

Cadogan, T. 2021. GoFundMe CEO: Hello congress, Americans need help and we can't do your job for you. USA Today. (Recuperado el 22 Junio 2021).

Elmer, G., Ward-Kimola, S., \& Burton, A. G. 2020. Crowdfunding during COVID-19: An international comparison of online fundraising. First Monday.

Farhoud, M., Shah, S., Stenholm, P., Kibler, E., Renko, M., \& Terjesen, S. 2021 . Social enterprise crowdfunding in an acute crisis. Journal of Business Venturing Insights, 15, e00211.

Farny, S., Kibler, E., Down, S. 2019. Collective emotions in institutional creation work. Academy of Management Journal, $62(3), 765-799$.

Igraa, M., Kenworthyb, N., Luchsingerc, C., Jungd, J.K. 2021. Crowdfunding as a response to COVID-19: Increasing inequities at a time of crisis. Social Science and Medicine (282).

Merelli, A. 2020. A record-breaking crowdfunding campaign is helping Italy fight Covid-19. En https:// qz.com/1836221/record-breaking-gofundmecampaigns are-helping-italy-fight-covid-19/. (Recuperado el 22 Junio 2021).

Rajwa, P., Hopen, P., Mu, L., Paradysz, A., Wojnarowicz, J., Gross, C. P., \& Leapman, M. S. 2020. Online Crowdfunding Response to Coronavirus Disease 2019. Journal of General Internal Medicine, 35, 2482-2484.

Renko, M., Moss, T.W., Lloyd, A. 2019. Crowdfunding by non-profit and social ventures. In: Landstrom, H., Parhankangas, A., Mason, C. (Eds.), Handbook of Research on Crowdfunding. Edward Elgar Publishing, Cheltenham, UK. 249-268.

Ryssdal, K., Fam, A. 2020. GoF undMe Sees Unprecedented Use during COVID-19 (Marketplace).

Saleh, Sameh Nagui, Lehmann, Christoph U., Medford \& Richard J. 2021. Early crowdfunding response to the COVID-19 pandemic: cross-sectional study. Journal of Medical Internet Research 23 (2), e25429 https://doi. org/10.2196/25429. 